Supplement of Arch. Anim. Breed., 63, 249-259, 2020

https://doi.org/10.5194/aab-63-249-2020-supplement

(c) Author(s) 2020. This work is distributed under

the Creative Commons Attribution 4.0 License.

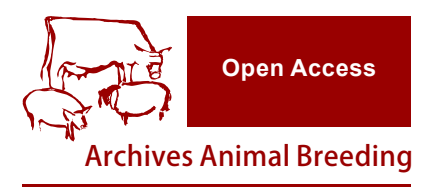

(c) (i)

Supplement of

\title{
Molecular characterization, tissue expression and polymorphisms of buffalo PPARGC1A gene
}

Lihua Qiu et al.

Correspondence to: Yongwang Miao (yongwangmiao1@126.com)

The copyright of individual parts of the supplement might differ from the CC BY 4.0 License. 
Table S1. Data information for tissue differential expression of buffalo PPARGC1A gene

\begin{tabular}{cccc}
\hline \multirow{2}{*}{ Tissues } & \multicolumn{2}{c}{ Relative expression level (mean \pm standard error)* } & \multirow{2}{*}{$P$ values } \\
\cline { 2 - 3 } & lactation & non-lactation & \\
\hline Muscle & $23.1816 \pm 0.9303$ & $39.3687 \pm 5.4650$ & 0.0432 \\
Heart & $18.3880 \pm 1.3240$ & $9.4338 \pm 0.8944$ & 0.0050 \\
Liver & $9.7518 \pm 0.2973$ & $5.3388 \pm 0.4343$ & 0.0011 \\
Brain & $6.4906 \pm 0.3496$ & $5.5206 \pm 0.1164$ & 0.0580 \\
Kidney & $2.8722 \pm 0.2056$ & $4.6320 \pm 0.5773$ & 0.1038 \\
Small intestine & $0.3704 \pm 0.0348$ & $0.9644 \pm 0.0181$ & 0.0001 \\
Mammary gland & $0.1404 \pm 0.0239$ & $0.6643 \pm 0.0404$ & 0.0004 \\
Rumen & $0.0558 \pm 0.0097$ & $0.4843 \pm 0.0293$ & 0.0002 \\
Spleen & $0.3268 \pm 0.0410$ & $0.0899 \pm 0.0204$ & 0.0066 \\
Lung & $0.0989 \pm 0.0166$ & $0.0150 \pm 0.0004$ & 0.0297 \\
\hline
\end{tabular}

$*$ The values are the average values for each tissue in four lactating or four non-lactating buffaloes. 
$\begin{array}{cccccc}10 & 20 & 30 & 40 & 50 & 60\end{array}$ eeeecct tchhhhhhhhhhhccccccccccccccccccccc hhhhhhhhcccccchhhhhhhcccc PSNIFEKIDEENEANLLAVLTETLDSLPVDEDGLPSFDALTDGDVTTENEASPSSMPDGTPPPQEAEEPS

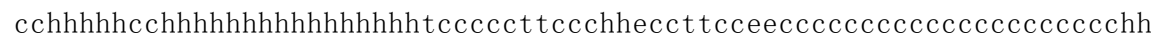
LLKKLLLAPANTQLSYNECSGLSTQNHANHNHRIRTNPAVVKTENSWSNKAKS ICQQQKPQRRPCSELLK hhhhhhhcccccceeechhtcccccccccccccccceeee hochhhhhhhhht tcccccchhhhhh YLTTNDDPPHTKPTENRNSSRDKCTSKKKAHTQSQTQHLQAKPTTLSLPLTPESPNDPKGSPFENKT IER

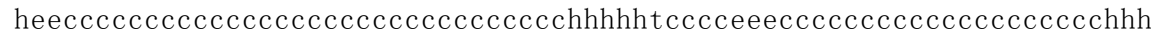
TLSVELSGTAGLTPPTTPPHKANQDNPFRASPKLKPSCKTVVPPPSKKARYSESSCTQGSNSTKKGPEQS eeeeettccccccccccccccccccccccttcceecccccceeecccccccccccccch ELYAQLSKTSVLTSGHEERKAKRPSLRLFGDHDYCQS INSKMEILVSTSQELHDSRQLENKDAPSSNGSG hhhhhhccceeeeccccc cсcc teeeeccchhhhhhhhheeehhhhhhhhhhccccccccccccc QIHSSTDSDPCYLRETAEVSRQVSPGSTRKQLQDQE IRAELNKHFGHPSQAVFDDKADKTSELRDSDFSN

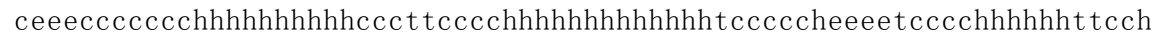
EQFSKLPMF INSGLAMDGLFDDSEDESDKLNSPWDGTQSYSLFHVSPSCSSFNSPCRDSVSPPKSLFSQR hhhhhcceeect thhhhhhhcccccchhhhccccccccceeeeecccccccccccccccccceehcc PQRMRSRSRSFSRHRSCSRSPYSRSRSRSPGSRSSSRSCYYYESGHCRHRTHRNSPLCARSRSRSPHSRR

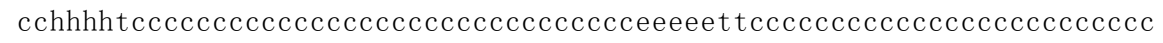
PRYDSYEEYQHERLKREEYRREYEKRESERAKQRERQRQKAIEERRVIYVGKIRPDTTRTELRDRFEVFG

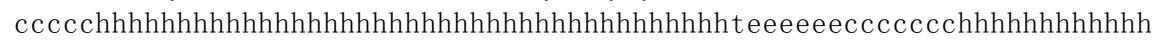
EIEECTVNLRDDGDSYGF ITYRYTCDAFAALENGYTLRRSNETDFELYFCGRKQFFKSNYADLDSNSDDF hhhhheeeeccttcceeeeeecchhhhhhhht tceeccccccheeeeetcceeet teeecccccccc DPASIKSKYDSLDFDSLLKEAQRSLRR cchhhccccchhhhhhhhhhhhhhhhh

Figure S1. Predicted secondary structure of buffalo PPARGC1A. Alpha helices, extended strands, beta turns, and random coils are indicated with the $\mathrm{h}, \mathrm{e}, \mathrm{t}$, and c, respectively. 